\title{
Influence of gaze direction on pointing to unseen proprioceptive targets
}

\author{
Annabelle Blangero ${ }^{1,2}$, Yves Rossetti ${ }^{1,2,3}$, Jacques Honoré ${ }^{4}$, Laure Pisella ${ }^{1,2}$ \\ ${ }^{1}$ Espace et Action, UMR INSERM Unité 534, Institut National de la Santé Et de la Recherche Médicale - Université \\ Claude Bernard Lyon I, 16 avenue Lépine, Case 13, 69676 Bron - France \\ ${ }^{2}$ Institut Fédératif des Neurosciences de Lyon: Hôpital Neurologique, Lyon- France \\ ${ }^{3}$ Service de Rééducation Neurologique, Hôpital Henry Gabrielle, Hospices Civils de Lyon, Route de Vourles, \\ St Genis Laval - France \\ ${ }^{4}$ Laboratoire de Neurosciences du Comportement, Université de Lille1, France
}

Received 17.06.2005

Accepted 22.11.2005

\section{Keywords}

Gaze, pointing, proprioceptive, retinal coordinates, sensori-motor

\section{ABSTRACT}

The question of how sensory information is encoded and integrated for goal-directed movements is a major topic in action research. Here we studied the influence of the direction of gaze on a task in which healthy individuals were required to point to their own unseen fingertip. An effect of the position of gaze on pointing, leading to pointing errors in the direction opposite to the gaze position, was obtained in the range of $11^{\circ}$ but vanished for $22^{\circ}$. These results suggest that targets of aiming movements performed with an unseen arm may be encoded in retinal coordinates even when the target is encoded in a nonvisual modality and remains unseen.

\section{INTRODUCTION}

In order to guide a goal-directed hand movement, the target has to be localised by at least one sensory system, and this information has to be ultimately transformed into an appropriate motor command. One of the main topics in action research is the nature of the reference frame that is used to represent the location of the target of an aiming movement. The classical view implies a sequential organisation of transformations that produces a representation of the target in a body-centred reference frame (Soechting \& Flanders, 1989a; Soechting \& Flanders, 1989b; Flanders, Tillery, \& Soechting, 1992; McIntyre, Stratta and Lacquaniti, 1998). In this model, the location of a visual target, initially coded in retinal coordinates, is combined with eye-position signals that give rise to the representation of the target in a head-centred reference frame. Then the position of the head is added to form a representation of the target location in body-centred coordinates. Finally the current position of the hand in body-centred coordinates is subtracted from the location of the target in body centred reference frame to obtain the motor vector in hand-centred coordinates. If the target is presented in another sensory modality, its coordinates enter in the chain of transformation processes at the level corresponding to the reference frame used in its specific modality (e.g. head level for auditory input). Since a proprioceptive target is coded in a body-centred reference frame, its location is integrated only during the later stage of processing, i.e. the position of the eyes should not affect the pointing movement.

An alternative model of visuo-motor integration, referred to as the direct method, has been proposed more recently (Buneo, Jarvis, Batista and Andersen, 2002). In this model, both the visual target and the initial position

Correspondence concerning this article should be addressed to Laure Pisella, Espace et Action, UMR-S INSERM U534, 16 avenue Lépine 69676 Bron - France. Electronic mail may be sent via Internet to pisella@lyon.inserm.fr 
of the hand are encoded in eye-centred coordinates. Then, the motor vector can be directly obtained by subtracting the location of the hand to the location of the target. This model is not supposed to be relevant when both the pointing hand and the target are not visible: "direct transformations may be the preferred scheme when both target location and the current hand position are simultaneously visible, even for a brief instant. In contrast, a sequential scheme may be used when visual information about the current position of the hand is unavailable" (Andersen and Buneo, 2002, p.208).

When the target is not visual, but from another sensory modality, is its position integrated into the sequential transformation processing (classical view) or is it systematically recoded in an eye-centred reference frame (generalisation of the retinal direct method to other sensory modalities)? As it has been shown that proprioceptive information is used to encode initial hand position when pointing to a visual target (Rossetti, Desmurget, \& Prablanc, 1995), the simplest way to implement a movement to a proprioceptive target would be to rely on a proprioceptivo-proprioceptive vector (i.e. a proprioceptive direct method). Hence no influence of gaze position should be observed when an unseen hand points toward an unseen target finger.

We investigated the potential influence of the direction of the gaze in a proprioceptive pointing task in healthy participants. In order to reduce the obvious bias toward visual coordinates when either the goal or the effector is encoded visually, we selected a task excluding informative visual inputs: pointing with the unseen hand to an unseen fingertip of the other hand.

\section{MATERIALS AND METHODS}

Eight naive subjects, between 24 and 40 years old, with normal or corrected to normal vision, participated in this experiment. Subjects remained naive about the purpose of the experiment. The task involved pointing to the left fingertip (proprioceptive target) with the right hand.

The subjects sat $30 \mathrm{~cm}$ in the front of a transparent vertical panel fixed upon a table. This pointing plane was placed such that it coincided with the subject's sagittal plane and hidden to their view by an opaque curtain. The curtain prevented the subject from seeing his/her two arms and forearms (no feed-back available), as well as the experimenter's eyes, so no cue about target location was available from vision (Fig1A). Seven target positions were located on a vertical line close to the proximal edge of the pointing plane. Only three experimental tar- gets were included in the analysis, the others were distractors preventing subjects from getting used to, and learning, the target positions. Among these three targets, the central one was set at the horizontal level of gaze $\left(0^{\circ}\right)$. The two other were positioned $6 \mathrm{~cm}$ above and below the central target $\left( \pm 11^{\circ}\right)$. The distractor targets were set at $\pm 2 \mathrm{~cm}, \pm 4 \mathrm{~cm}$ and $\pm 8 \mathrm{~cm}$ around the central target (corresponding to $\pm 3.67^{\circ}, \pm 7.33^{\circ}$ and $\left.\pm 14.67^{\circ}\right)$. Another vertical array of seven coloured dots of about 1.5 degrees of visual angle was visible to the subject (on the curtain), on the edge of the vertical panel, so that each dot was located at the same height as a target position. These coloured dots were used as fixation points. As for the pointing targets, only three coloured dots were used for the analysis $(-6 \mathrm{~cm}, 0 \mathrm{~cm}$ and $+6 \mathrm{~cm})$, the others served as distractors. Therefore the unseen proprioceptive targets could be presented at 0,11 or $22^{\circ}$ of visual angle up or down from gaze direction (Fig1A).

The left index fingertip was passively placed by the experimenter on the left side of the pointing plane and the task was to point with the right index toward the left finger, by touching the vertical plane in the most accurate position (with no speed constraint). The pointing plane was thick enough to prevent any tactile-tactile feedback. No constraint was imposed on the pointing-arm posture. It turned out that all subjects used a standard comfortable posture when pointing (Rossetti, Meckler, \& Prablanc, 1994). The starting positions used for the two hands were different in order to prevent the subjects from performing their pointing responses by simply reproducing the passive movement of the left arm (Rossetti et al., 1995) (Fig1B). After a few training trials, subjects were asked to perform another task simultaneously: subjects were verbally instructed to fixate one of the coloured dots. The subjects were told that this additional colour-fixation task was distracting with respect to the main proprioceptive pointing task. After each fixation instruction, their left index finger was passively positioned on one target, and they had to point at it with their right hand (Fig1). Care was taken to keep the delay between eye fixation and the end of the trial below 2 seconds, in order to limit possible eye position adaptation effects. Furthermore, a second experimenter checked that subjects maintained eye position throughout each trial. Target position and fixation point were varied independently and the trial selection followed a predetermined pseudo-random order. Each experimental target was presented 24 times. The other (distractor) targets were presented 4 or 8 times each. 


\section{A - Experimental set-up}

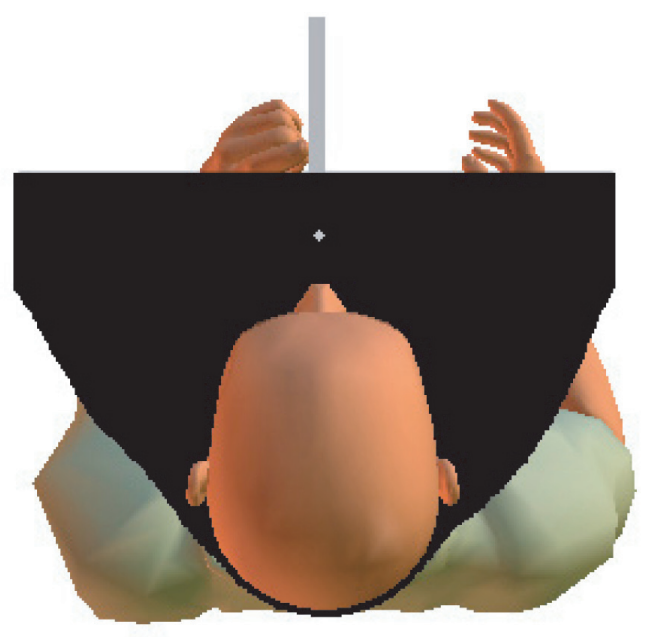

Top View

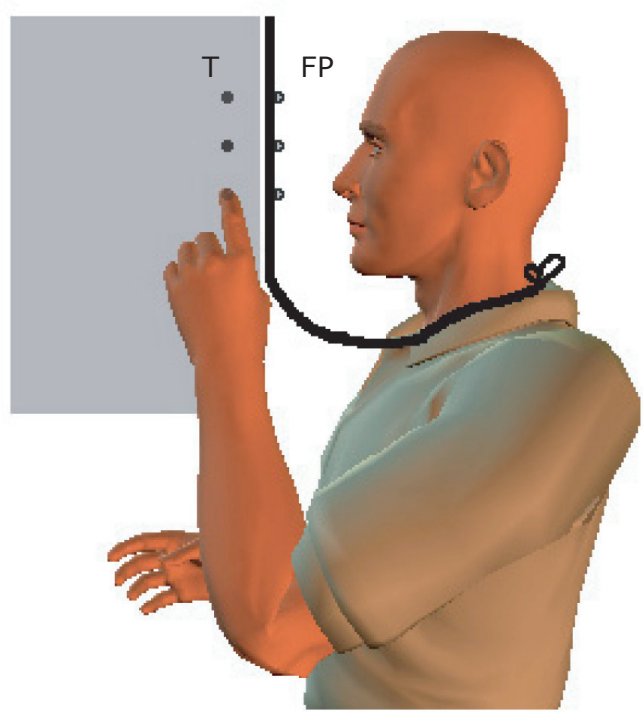

Side View

B - Experimental procedure

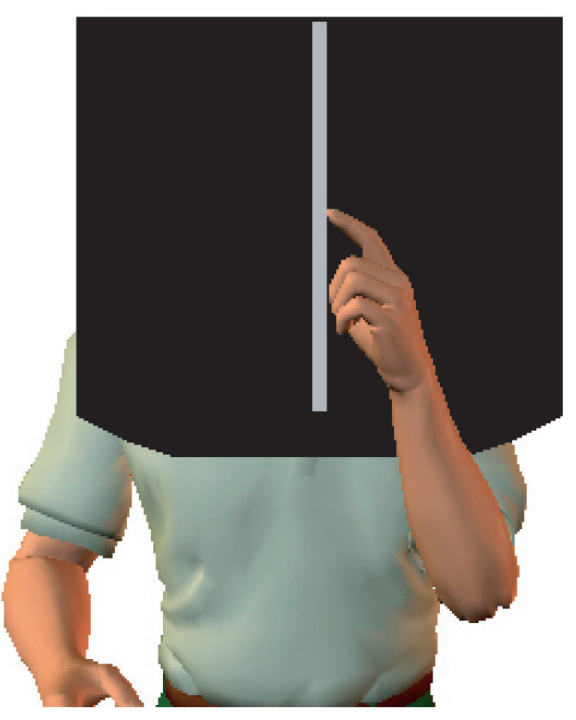

Passive Positioning

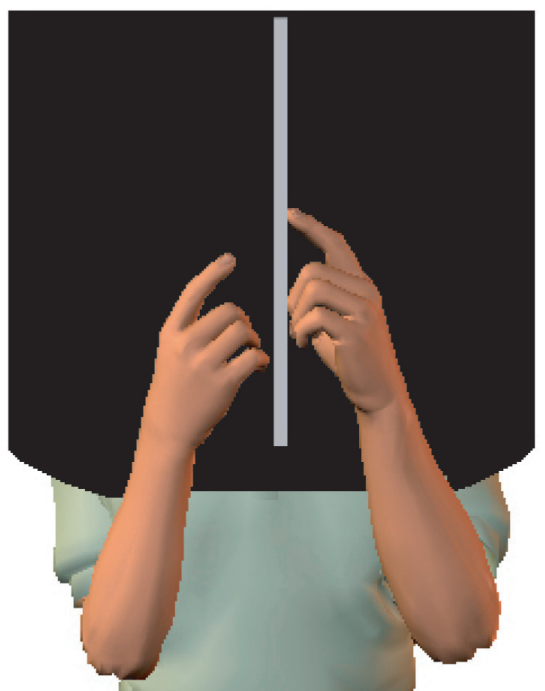

Active Pointing

\section{Figure 1}

The subject sat at $30 \mathrm{~cm}$ from an opaque curtain. Behind it there was a vertical screen that coincided with the subject sagittal plan (A). In each trial the subject was instructed to visually fixate one of the three coloured dots, and then the left index (target) was passively positioned by the experimenter behind the curtain at one of the three possible locations (A). It could be the same as the fixation location or a different one. The task was then to point with the right index to the felt position of the left fingertip without seeing it (B).

For each trial, the pointing error was measured as the vertical difference between the target position (left fingertip) and the right fingertip end-point, measured by a milli- metric ruler fixed on the panel. Errors were signed positive when endpoints were higher than the targets and signed negative when endpoints were lower than the targets. 
Eye position main effect

$Y=-7,5225+0,5081 * X$

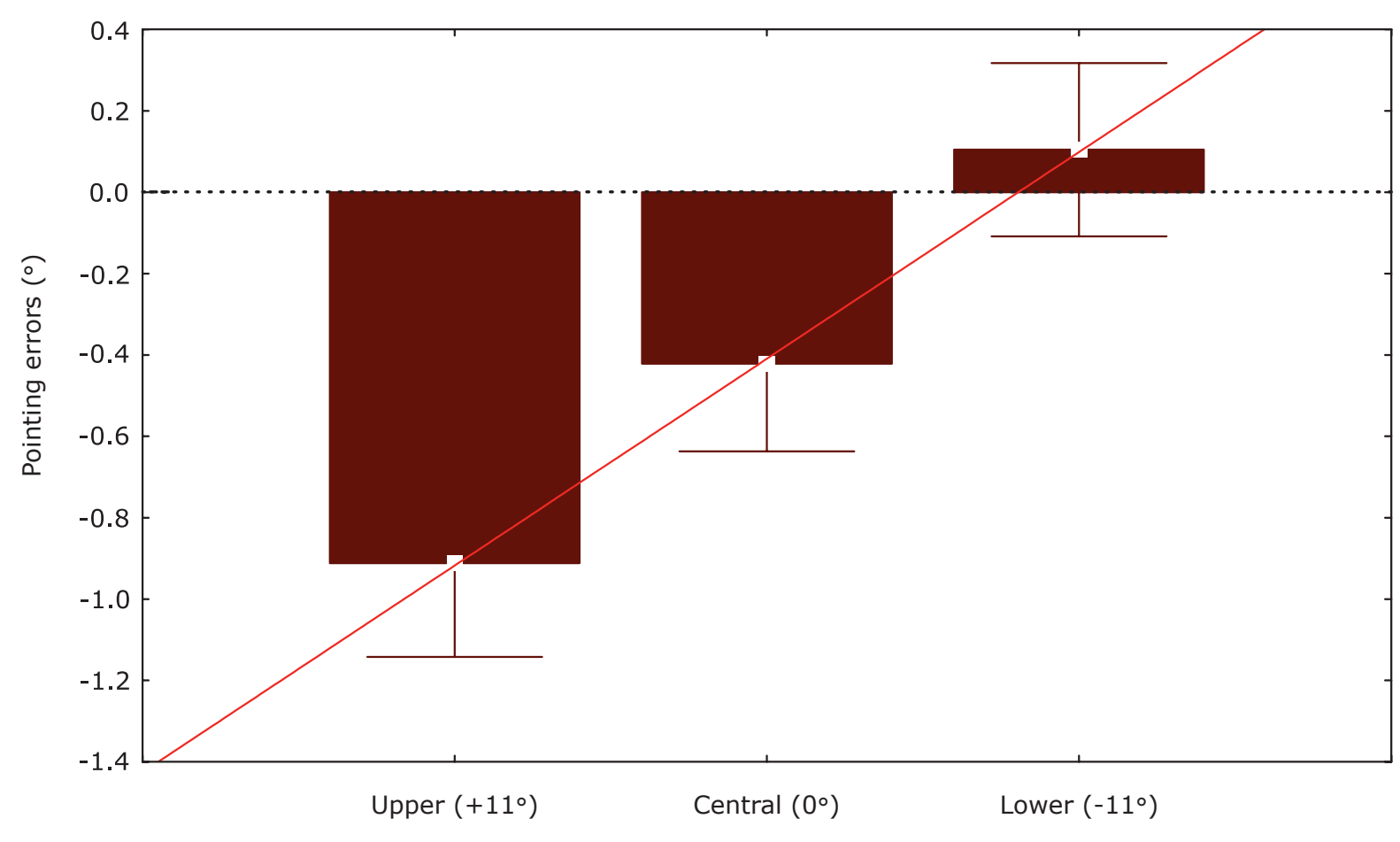

Eye Position ( $\left.{ }^{\circ}\right)$

Figure 2

Means of all pointing endpoint errors for the three locations of eye fixation (upper, central, lower). This graph is an illustration of the main effect of the eye position on pointing errors. This main effect indicates pointing errors in the direction opposite to the gaze position.

\section{RESULTS}

In a first analysis, the Eye and the Target main effects on pointing accuracy could be distinguished from the nine combinations of eye position and target position (3 Eye $\times 3$ Target). A repeated measures ANOVA was computed with the individual mean errors obtained for each of the nine combinations. Surprisingly, the target main effect did not reach significance $(F[2,14]=0.02 ; p>.98)$. By contrast, the eye main effect was significant $(F[2,14]=11.825 ; p<.001)$ even though the pointing task included no view of either the target finger or the pointing finger. Figure 2 shows how the direction of the gaze influenced pointing behaviour away from fixation.

The Eye by Target interaction was also significant $(F[4,28]=9.92 ; p<.001)$. In order to explore this interaction effect, we categorized the data by rearranging them in terms of the interval size between gaze and the position of the proprioceptive target (that we will call a gap). A total of five eye-target gap values were obtained: $-22,-11,0,+11$ and +22 visual degrees. A positive gap means that the eyes were positioned vertically above the target. In order to focus on the influence of gaze and to eliminate the inter-individual constant bias linked with proprioceptive pointing, we normalized the data by subtracting the errors obtained in the no gap condition $\left(0^{\circ}\right)$ from the errors obtained for the different gaps. Figure $3 a$ illustrates the pointing errors relative to the different gaze-target gaps for each individual subject after normalization.

Despite important inter-individual variability, these graphs show a common qualitative pattern of influence of gaze direction on the pointing behaviour of our subjects, especially for the eye-target gaps of $-11^{\circ}$ and $+11^{\circ}$ : gaze position produces a pointing bias opposite to the direction of gaze. This is confirmed by the t-tests performed on the group mean values (Fig 3B), showing that the errors deviate significantly from zero for a gap of $-11^{\circ}(t[7]=3.25 ; p<.05)$ as well as for a gap of $+11^{\circ}$ $(t[7]=2.63 ; p<.05)$. By contrast, errors were not statistically different from zero for a gap of $-22^{\circ}(t[7]=-1.21$; $p>.26)$ and for a gap of $+22^{\circ}(t[7]=-0.95 ; p>.37)$. Therefore, the effect of the gaze direction on proprioceptive pointing is only significant for a difference of $11^{\circ}$ between the eyes and the finger, bu $t$ not for a gap of $22^{\circ}$. The effect of gaze direction faded at $\pm 22^{\circ}$, probably because of a larger inter-individual variability. 
All subjects pointing errors for the different eye-target gaps

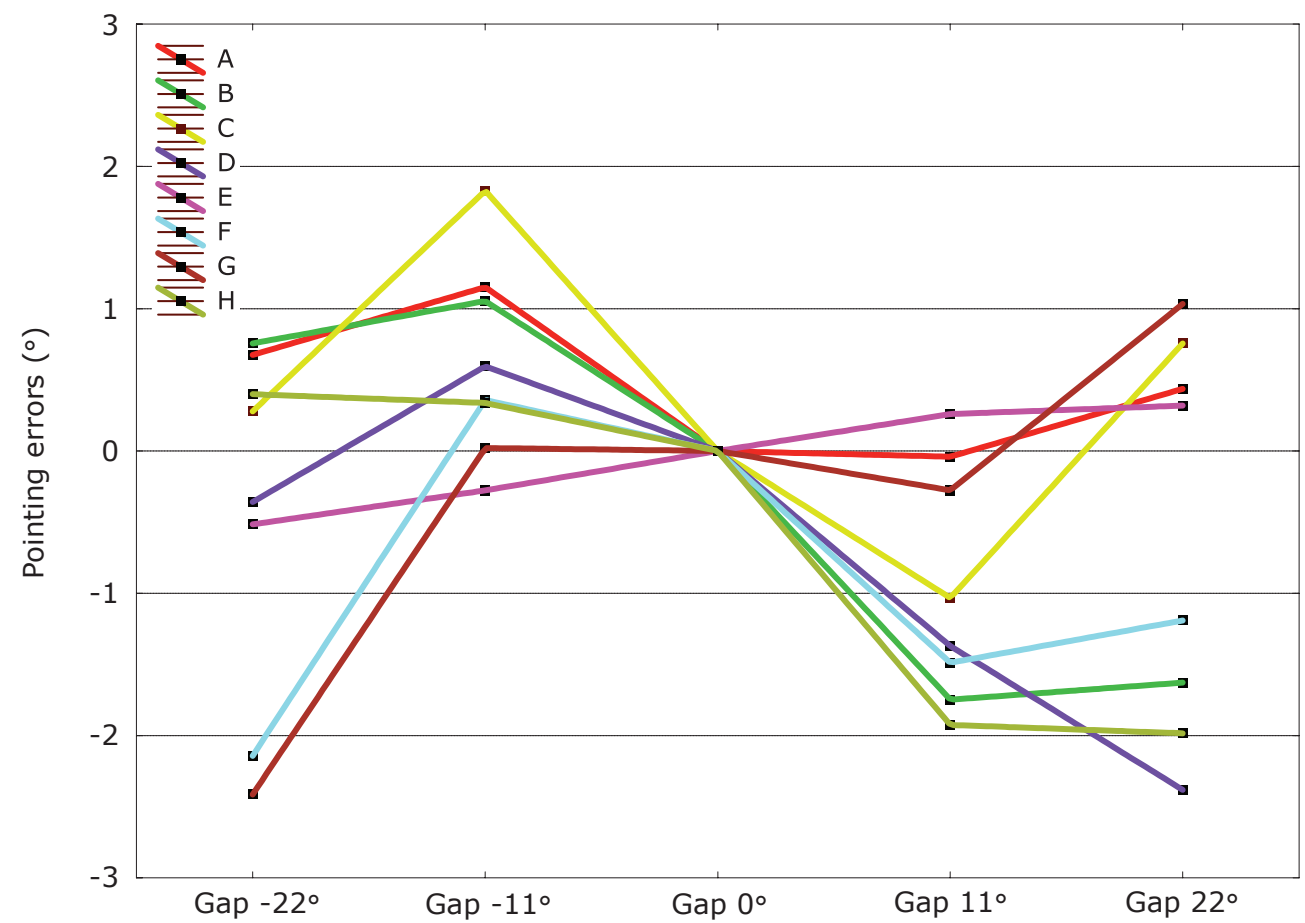

Eye Target Gap $\left({ }^{\circ}\right)$

\section{Figure 3a}

Means of pointing errors normalized for every subject for the different eye-target gap $\left(-22^{\circ},-11^{\circ}, 0^{\circ}+11^{\circ},+22^{\circ}\right)$. This graph is an illustration of the interaction between the eye position and the target position.

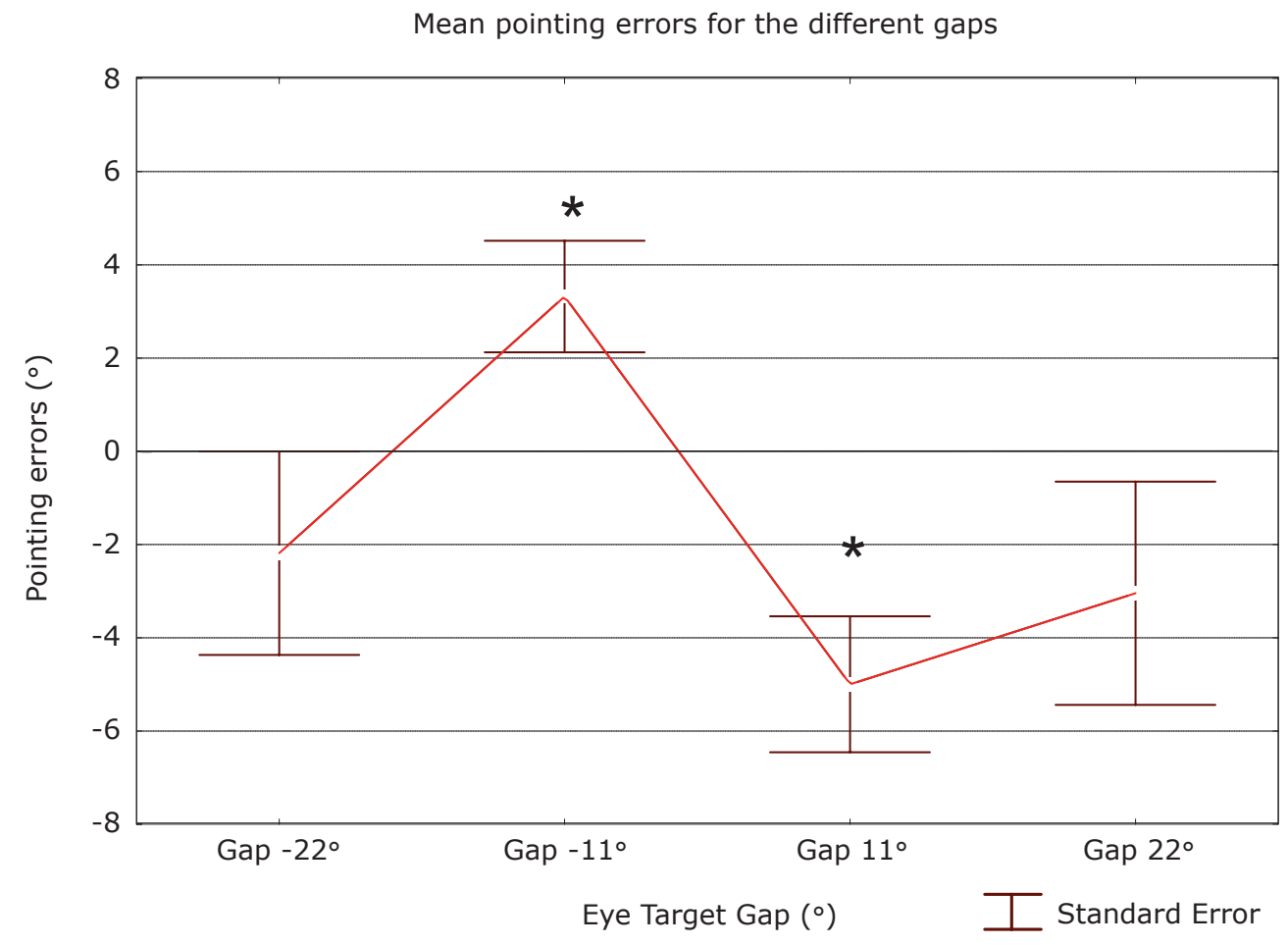

Figure $3 \boldsymbol{b}$

Means of pointing errors normalized for all subjects for the different eye-target gap $\left(-22^{\circ},-11^{\circ}, 0^{\circ}+11^{\circ}\right.$, $\left.+22^{\circ}\right)$. The errors were significantly different from zero for the $-11^{\circ}$ and $+11^{\circ} \mathrm{gap}$. 


\section{DISCUSSION}

The present study aimed to investigate whether gaze position influences the sensori-motor transformation of non-visual target location. The results will be discussed in three steps. First, we showed an influence of gaze direction on movements planned toward a proprioceptive target i.e., a target which is not encoded primarily through the visual sensory system. Second, this "visual" effect on proprioceptive pointing is characterised by pointing errors in the direction opposite to the gaze position. Third, this effect was not significant at $22^{\circ}$ of visual eccentricity, and is thus limited to the near-foveal vision. These points will be successively discussed in the light of the two hypotheses presented in introduction: the sequential model and the direct method of sensori-motor transformation.

According to the sequential model (Soechting \& Flanders, 1989a; Soechting and Flanders, 1989b; Flanders et al., 1992; McIntyre et al., 1998), proprioceptive targets enter the coordinate transformation process directly at the stage when a representation is formed based on a body-centred reference frame, hence no influence of gaze position should have been obtained. However, our study demonstrated an influence of gaze direction on pointing with an unseen arm toward unseen proprioceptive targets. This clearly demonstrates that direct proprioceptivo-proprioceptive coding was not used during this task. Several studies have already reported that pointing is influenced by visual information about the pointing arm available prior to movements made to visual (Rossetti et al., 1995; Rossetti et al., 1994) or proprioceptive (Desmurget, Rossetti, Jordan, Meckler, \& Prablanc, 1997) targets. Their conclusion was that both visual and proprioceptive cues about initial hand position were used (Rossetti at al., 1995). In our experimental conditions, neither the initial nor the in-flight hand position was visible. Even the direct method as initially described by Andersen and Buneo (2002, p.208) would have predicted that this total lack of visual guidance should have prevented movement planning to be executed with reference to visual coordinates. However, an influence of gaze direction had been also demonstrated in visual open-loop conditions (absence of hand visual feedback) for immediate (Bock, 1986; Bock, 1993) or delayed (Enright, 1995) pointing to visual targets, and when pointing toward remembered visual targets following an eye movement (Henriques, Klier, Smith, Lowy, \& Crawford, 1998; Henriques \& Crawford, 2000; Khan,
Pisella, Vighetto et al. 2005). These results already suggested an extension of the sensori-motor transformation described by the direct method to conditions in which effector and/or target are not visible during the period of movement planning. The results of the present experiment further extend this mode of sensori-motor transformation to targets in other modalities, since a proprioceptive target was used, ensuring that the encoding of its position is not retinal. Taken together, these results suggest that oculocentric coordinates may contribute to a pointing task even when it is performed entirely in the absence of relevant visual information. Such visual influence has been previously shown for auditory targets (Lewald, 1998; Lewald \& Ehrenstein, 1996), but it is much more counterintuitive to reach the same conclusion with a proprioceptive pointing task that could be simply performed in body-coordinates.

Second, the present results show that pointing movements were shifted in the direction opposite to the gaze position. Several visual and auditory pointing experiments performed on humans with an imposed eye fixation (Bock, 1986; Bock, 1993; Enright, 1995; Henriques et al., 1998; Henriques \& Crawford, 2000; Lewald, 1998; Lewald \& Ehrenstein, 1996; Pouget, Ducom, Torri, \& Bavelier, 2002) have described a similar directional effect, i.e. errors opposite to gaze position. However, only Bock (Bock, 1986, 1993), in his studies of visual pointing, provided an explanation of the direction of this effect, proposing that it may result from the retinal magnification factor. According to Bock, a visual stimulus seen in the peripheral visual field is perceived as being further from eye fixation than it really is. It could then be speculated that all sensory representations are remapped onto this visual representation to guide action. Consequently, the magnification factor could be observed for the localisation of targets presented in other modalities. Indeed, it should be noted that this direction of errors is opposite to what would be predicted from a simple "range effect". Since only this retinal factor has been proposed to account for the direction of influence of gaze on the endpoints, a "visual representation" of the target (including the retinal magnification) may be built up even in the absence of relevant retinal input. Such a mechanism would explain why pointing errors have been consistently found in the direction opposite to the foveal position not only for visual targets, but also for auditory and proprioceptive targets. This hypothesis is compatible with a common eye-centred representation of space used for planning goal directed movements (Cohen \& Andersen 2000, 2002). 
The third present result is that the effect of gaze position on pointing was obtained in the range of $11^{\circ}$ but disappeared for eccentricity of $22^{\circ}$. This is consistent with results obtained with visually guided pointing, which indicate that the effect of gaze position tends to decrease beyond eccentricities of $20^{\circ}$ (Bock, 1986; Bock, 1993; Henriques et al., 1998; Henriques \& Crawford, 2000). This similar pattern of influence of gaze direction on pointing toward visual and non-visual targets is an additional argument for the hypothesis that targets locations from other sensory modalities may be adjusted into an eye-centred representation, and thus be subjected to the retinal magnification factor. Note that the amplitude of the effect and its eccentricity range appears less important for proprioceptive and auditory targets than for visual targets (Lewald, 1998; Lewald \& Ehrenstein, 1996; Pouget et al., 2002). Therefore additional processes necessary to transform target locations from the initial sensory modality to the eye-centred representation tend to reduce the amplitude of the eye-position effect.

Finally, the two models described in the introduction can be modified in order to account for the present results. In the context of the sequential model, it can be postulated that gaze direction modifies the representation of the location of the target, at any stage of processing, by a kind of gain modulation mechanism. This updated model would then not be purely sequential. The present results also cannot be accounted for by the model of the direct method as it was initially presented (Buneo et al., 2002; Andersen \& Buneo, 2002). Indeed, it postulated a representation of the target in an eye-centred reference frame only when the hand and the target were visible (closed-loop). In order to account for many of the results reviewed above, including the present ones, the direct method could be extended both to open-loop pointing conditions and to targets encoded in other sensory modalities. This implies that target locations from different sensory modalities would be represented in a common eye-centred reference frame. According to this view, an electrophysiological study on the macaque's posterior parietal cortex (Cohen \& Andersen, 2000, 2002) showed that the same neurons of the parietal reach region respond to a visual or an auditory target, and that both targets are encoded in an eye-centred reference frame. Nevertheless, this eye-centred planning is probably not the only mode, but it is rather likely that different representations can coexist and interact in the brain to plan a single pointing movement.

\section{CONCLUSION}

Our experiment revealed an influence of gaze on pointing toward an unseen proprioceptive target. This result is not compatible with the classical view of a sequential transformation of the sensory input into a motor command. We postulate the existence of a common, multisensorial representation of space in an eye-centred reference frame. This hypothesis is consistent with recent findings from electrophysiological studies on the monkey's parietal reach region (Cohen \& Andersen, 2000, 2002).

\section{References}

Andersen, R.A., Buneo, C.A. (2002). Intentional maps in posterior parietal cortex. Annual Review of Neuroscience, 25, 189-220. | $\underline{\mathrm{wWw}}$

Bock, O. (1986). Contribution of retinal versus extraretinal signals towards visual localization in goal-directed movements. Experimental Brain Research, 64, 476-482. Www

Bock, O. (1993). Localization of objects in the peri-pheral visual field. Behavioural Brain Research, 56: , 77-84. |ww|

Buneo, C.A., Jarvis, M.R., Batista, A.P., Andersen, R.A. (2002). Direct visuomotor transformations for reaching. Nature, 416, 632-636. $\mid \underline{w w \mid}$

Cohen, Y.E., Andersen, R.A. (2000). Reaches to sounds encoded in an eye-centred reference frame. Neuron, 27, 647-652. Www

Cohen, Y.E., Andersen, R.A. (2002). A common reference frame for movement plans in the posterior parietal cortex. Nature Reviews Neuroscience, 3, 553-562. $\underline{w w w}$

Desmurget, M., Rossetti, Y., Jordan, M., Meckler, C., Prablanc, C. (1997). Viewing the hand prior to movement improves accuracy of pointing performed toward the unseen contralateral hand. Experimental Brain Research, 115, 180-6. |Www

Enright J.T. (1995). The non-visual impact of eye-orientation on eye-hand coordination. Vision Research, 35, 1611-1618. $\underline{\underline{w W} \mid}$

Flanders, M., Helms Tillery S.I., Soechting J.F. (1992). Early stages in a sensorimotor transformation. Behavioral and Brain Sciences, 15, 309-362. Www

Henriques, D.Y.P., Crawford, J.D. (2000). Direction-dependent distortions of retinocentric space in the visuomotor transformation for pointing. Experimental Brain Research, 132, 179-194. Www

Henriques, D.Y.P., Klier, E.M., Smith M.A., Lowy D., Crawford, J.D. (1998). Gaze-centred remapping of remembered visual space in an open-loop pointing task. Journal of Neuroscience, 18, 1583-1594. [Www 
Khan A.Z., Pisella L., Vighetto A., Cotton F., Luauté J., Boisson D., Salemme R, Crawford J.D., Rossetti Y. (2005). Optic Ataxia errors depend on remapped, not viewed, target location. Nature Neuroscience, 8(4), 418-420.

Lewald, J. (1998). The effect of gaze eccentricity on perceived sound direction and its relation to visual localization. Hearing Research, 115, 206-216. [Www

Lewald, J., Ehrenstein, W.H. (1996). Auditory-visual shift in localization depending on gaze direction. Neuroreport, 7, 1929-1932. |WWw

McIntyre, J., Stratta, F., Lacquaniti F. (1998). Shortterm memory for reaching to visual targets: psychophysical evidence for body-centred reference frames. Journal of Neuroscience, 18, 8423-8435. WwW

Pouget, A., Ducom, JC., Torri, J., Bavelier, D. (2002).
Multisensory spatial representations in eye-centred coordinates for reaching. Cognition, 83, B1-B11. |WWW Rossetti, Y., Desmurget, M., Prablanc, C. (1995). Vectorial coding of movement: vision, proprioception, or both? Journal of Neurophysiology, 74, 457-63. Www Rossetti,Y., Meckler, C., Prablanc, C. (1994). Is there an optimal arm posture? Deterioration of finger localization precision and comfort sensation in extreme arm-joint postures. Experimental Brain Research, 99, 131-6. [WwW

Soechting, J.F, Flanders M. (1989a). Errors in pointing due to approximations in sensorimotor transformations. Journal of Neuroscience, 62, 595-608. [www

Soechting, J.F, Flanders M. (1989b). Sensorimotor representations for pointing to targets in three-dimensional space. Journal of Neuroscience, 62, 582-594. Www 